\title{
Hybrid Linear/Quadratic Time-Frequency Attributes
}

\author{
Richard G. Baraniuk, Senior Member, IEEE, Mark Coates, Member, IEEE, and Philippe Steeghs
}

\begin{abstract}
We present an efficient method for robustly calculating time-frequency attributes of a signal, including instantaneous mean frequency, bandwidth, kurtosis, and other moments. Most current attribute estimation techniques involve a costly intermediate step of computing a (highly oversampled) two-dimensonal (2-D) quadratic time-frequency representation (TFR), which is then collapsed to the one-dimensonal (1-D) attribute. Using the principles of hybrid linear/quadratic time-frequency analysis (time-frequency distribution series), we propose computing attributes as nonlinear combinations of the (slightly oversampled) linear Gabor coefficients of the signal. The method is both computationally efficient and accurate; it performs as well as the best techniques based on adaptive TFRs. To illustrate, we calculate an attribute of a seismic cross section.
\end{abstract}

Index Terms-Gabor transform, instantaneous frequency, seismic signal analysis, time-frequency analysis.

\section{INTRODUCTION}

$\mathbf{T}$ IME-FREQUENCY signal attributes have provided insights in a number of fields, from seismic analysis to neurophysiology [1]-[3]. Attributes applied to date include instantaneous frequency, bandwidth, and kurtosis (normalized fourthorder instantaneous frequency moment) [4]. The instantaneous frequency (IF) of a real monocomponent signal is defined as the rate of change of the phase of the corresponding analytic signal at a given time. This is equivalent to the first moment in frequency of the Wigner distribution (WD) of the signal normalized by its instantaneous energy. Unfortunately, when we estimate the IF either directly from the phase of the analytic signal or from the $\mathrm{WD}$, we generate estimates with high variance, particularly in noisy environments [5]. Estimates based on the first moment in frequency of other time-frequency representations (TFRs) - the spectrogram, for instance-reduce the variance at the expense of some bias.

The peak of the WD or another suitable TFR offers another attractive alternative for IF estimation. The variance of an estimate based on TFR peaks is reduced compared with moment-based methods, especially in low SNR environments. In particular, iterative methods involving repeated calculation of TFRs have proven extremely effective [5]-[7].

Manuscript received June 13, 2000; revised December 27, 2000. This work was supported by the National Science Foundation under Grants MIP-9457438 and CCR-9973188, the Office of Naval Research under Grant N00014-99-1-0813, the Defense Advanced Research Projects Agency under Grant F49620-97-1-0513, Texas Instruments, and the Rice Consortium on Computational Seismic Interpretation. The associate editor coordinating the review of this paper and approving it for publication was Dr. Xiang-Gen Xia.

R. G. Baraniuk and M. Coates are with the Department of Electrical and Computer Engineering, Rice University, Houston, TX 77005-1892 (e-mail: richb@ece.rice.edu; mcoates@ece.rice.edu).

P. Steeghs is with TNO Physics and Electronics Laboratory, The Hague, The Netherlands (e-mail: steeghs@fel.tno.nl).

Publisher Item Identifier S 1053-587X(01)02259-0.
There are instances, however, when peak-based estimates are unsatisfactory. When multiple components are present in a signal, it is more desirable to calculate the moment-based IF estimate. Such is the case in the seismic analysis presented in Fig. 1 and Section V. We then need a high-resolution, signal-adaptive TFR to make the IF estimate useful. Unfortunately, generating such a TFR is computationally wasteful, since we merely collapse the 2-D TFR to a 1-D attribute.

In this paper, we present a computationally efficient method for estimating moment-based time-frequency attributes that avoids the calculation of a quadratic TFR altogether. Our approach exploits the time-frequency distribution series concept developed by Qian et al. [8]-[10] and requires only a sparse linear signal decomposition. We will present a general procedure for calculating a range of attributes but focus on IF and instantaneous bandwidth estimation for concreteness.

Section II reviews time-frequency distribution series, and Section III examines IF and its estimation. Section IV presents the new estimation technique for IF and instantaneous bandwidth, and Section V discusses its application to seismic data analysis. We make some concluding remarks in Section VI.

\section{Hybrid TIME-FREQUENCY REPRESENTATIONS}

Qian et al. [8], [9] proposed a method for time-frequency analysis that utilizes both a linear TFR (the Gabor transform) and a quadratic TFR (the WD) to generate a signal adaptive nonlinear TFR. The Gabor transform decomposes a signal $x(t)$ in terms of logons [11] or time-frequency atoms

$$
\begin{aligned}
x(t) & =\sum_{(m, n)} c_{m, n} \phi_{m, n}(t), \quad(m, n) \in \mathbb{Z}^{2} \\
c_{m, n} & :=\left\langle x, \tilde{\phi}_{m, n}\right\rangle:=\int_{-\infty}^{\infty} x(t) \tilde{\phi}_{m, n}^{*}(t) d t .
\end{aligned}
$$

The synthesis atoms are generated by time-frequency shifting a prototype atom $g(t)$ by discrete step sizes $T$ and $F$

$$
\phi_{m, n}(t):=e^{j 2 \pi m F t} g(t-n T) .
$$

The $\tilde{\phi}$ are dual atoms derived from $\phi$ [8], [11]. A natural choice for the prototype atom $g$ is the Gaussian, since it has optimal concentration and localization in time-frequency and a strictly positive WD [12]. In order for (1) to be a stable representation with Gaussian atoms requires a mild oversampling in the time-frequency plane.

The auto-WD of a signal $x(t)$ is defined as

$$
W_{x}(t, f):=\int_{-\infty}^{\infty} x(t+\tau / 2) x^{*}(t-\tau / 2) e^{-j 2 \pi f \tau} d \tau .
$$



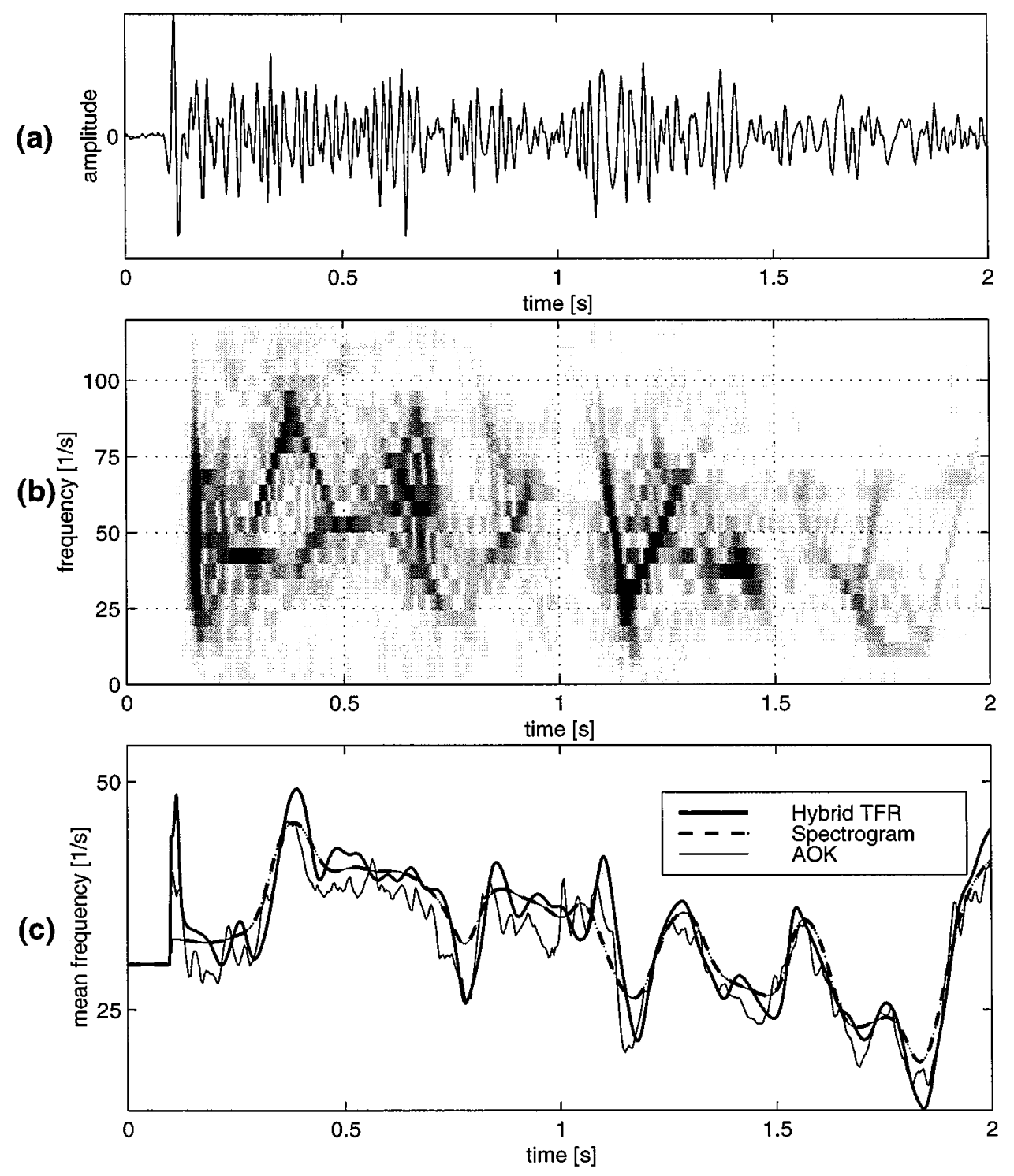

Fig. 1. Instantaneous frequency (IF) estimation of a seismic recording. (a) Seismic data. (b) Adaptive time-frequency representation (TFR) [12] of the seismic trace. (c) Normalized instantaneous frequency estimates. Thick line is the hybrid IF estimate (Manhattan distance threshold $\delta=2$ ), thin line the adaptive TFR estimate, and dashed line the spectogram estimate. The hybrid IF estimate generated using no cross-terms (not shown) is very similar to the spectrogram estimate.

The cross-WD between two signals $x(t)$ and $y(t)$ is defined as

$$
W_{x y}(t, f):=\int_{-\infty}^{\infty} x(t+\tau / 2) y^{*}(t-\tau / 2) e^{-j 2 \pi f \tau} d \tau
$$

Inserting (1) in (4) and using (5), the auto-WD can be decomposed as

$$
\begin{aligned}
W_{x}(t, f)= & \sum_{(m, n)}\left|c_{m, n}\right|^{2} W_{\phi_{m, n}}(t, f) \\
& +\sum_{(m, n) \neq\left(m^{\prime}, n^{\prime}\right)} c_{m, n} c_{m^{\prime}, n^{\prime}}^{*} W_{\phi_{m, n}}, \phi_{m^{\prime}, n^{\prime}}(t, f) .
\end{aligned}
$$

This expression identifies two distinct contributions to the WD. The first summation in (6) corresponds to a linear sum of the (strictly positive) auto-WDs of the time-frequency atoms; it provides an approximate description of the signal's time-frequency behavior without capturing many details. The second summation involves all of the cross-WDs between different atoms in the Gabor decomposition. The cross-WDs in this summation may be positive or negative.

The key observation is this [8], [9]: The cross-WDs between closely spaced $\left[(m, n)\right.$ close to $\left.\left(m^{\prime}, n^{\prime}\right)\right]$ atoms generally refine the TFR of the signal, whereas the cross-WDs between distant $\left[(m, n)\right.$ far from $\left.\left(m^{\prime}, n^{\prime}\right)\right]$ atoms generate global interference terms that hamper interpretation.

Qian et al. generate a time-frequency distribution series-which we term a hybrid TFR [10]-by retaining all of the auto-WD terms in the first summation of (6) but only those cross-WDs arising from closely spaced atoms. Due to the elliptical or circular symmetry of the Gaussian atom, the Euclidean distance metric is the most natural measure of atom separation; the $l_{1}$ metric (Manhattan distance) $d\left[(m, n),\left(m^{\prime}, n^{\prime}\right)\right]:=\left|m-m^{\prime}\right|+\left|n-n^{\prime}\right|$ is a good approximation with a lower computational complexity. Given a 
threshold distance $\delta$, second summation terms are included in the final representation only if the distance between the interacting atoms is less than $\delta$. The hybrid method thus produces a $\delta$-parameterized class of TFRs

$$
\begin{aligned}
& \tilde{W}_{x}^{(\delta)}(t, f):=\sum_{(m, n)}\left|c_{m, n}\right|^{2} W_{\phi_{m, n}}(t, f) \\
& +\sum_{0<d\left[(m, n),\left(m^{\prime}, n^{\prime}\right)\right]<\delta} c_{m, n} c_{m^{\prime}, n^{\prime}}^{*} W_{\phi_{m, n}, \phi_{m^{\prime}, n^{\prime}}}(t, f)
\end{aligned}
$$

The parameter $\delta$ controls the tradeoff between component resolution and interference. By a suitable selection of $\delta$, high-resolution TFRs are generated; moreover, they can be determined at a much lower computational expense than signal-adaptive quadratic TFRs [13] offering similar performance. The auto- and cross-WDs in (7) are signal-independent and, hence, can be analytically computed and stored in memory. The computational demands of the Gabor transform are also much less than those of quadratic TFRs. By replacing the Gabor decomposition with a wavelet transform, we can generate high-resolution, low-interference time-scale representations [10].

\section{INTANTANEOUS FREQUENCY AND INSTANTANEOUS BANDWIDTH}

The instantaneous amplitude $a(t)$ and IF $v(t)$ of a real-valued signal $x(t)$ are defined in terms of the analytic signal $z_{x}(t)$ [12], [14], [15] as

$$
\begin{aligned}
a(t) & :=\left|z_{x}(t)\right| \\
v(t) & :=\frac{1}{2 \pi} \frac{d}{d t} \arg z_{x}(t) .
\end{aligned}
$$

For signals observed in noise, one means of estimating the IF of a discrete signal is immediately clear. We can apply (8) directly to the observed signal; we call this the complex-trace IF estimate. Unfortunately, such an estimate is extremely susceptible to noise.

More robust estimates can be formed by considering the relationship between the WD and the IF [12], [15]:

$$
v(t)=\frac{\int_{-\infty}^{\infty} f W_{x}(t, f) d f}{\int_{-\infty}^{\infty} W_{x}(t, f) d f}
$$

Direct utilization of this formula again leads to high-variance estimates in noisy environments. However, bias can be traded against variance by replacing the WD with a smoother TFR - the spectrogram, for instance. To obtain a good tradeoff and robustly track the IFs of signals whose time-frequency behavior is unknown and variable, we generally have to calculate a computationally intensive adaptive TFR [13]. Whichever TFR is chosen, considerable computational effort is wasted since we generate a highly redundant description of the 2-D, time-frequency nature of the signal merely to collapse back to the 1-D quantity $v(t)$.
The bandwidth of a signal at a particular moment in time (instantaneous bandwidth [14]) is closely related to the secondorder frequency moment of a TFR $P_{x}$ of the signal

$$
B_{P}^{2}(t)=\frac{\int_{-\infty}^{\infty} f^{2} P_{x}(t, f) d f}{\int_{-\infty}^{\infty} P_{x}(t, f) d f}-v(t)^{2} .
$$

As the resolution of the TFR improves, its second-order moment provides a better indication of instantaneous bandwidth. However, interference terms and noise make bandwidth estimates based on the high-resolution WD unstable.

\section{HYBRID INSTANTANEOUS FREQUENCY AND BANDWIDTH Calculation}

In this section, we detail how the hybrid TFR approach of Section II can be adopted to robustly estimate the IF and instantaneous bandwidth. Our approach requires the much smaller computational expense of determining a linear, mildly oversampled Gabor representation of the signal.

Substituting the Gabor expansion of the signal (1), we can rewrite the numerator of (9) as

$$
\sum_{\left(m, n, m^{\prime}, n^{\prime}\right)} c_{m, n} c_{m^{\prime}, n^{\prime}}^{*} \int_{-\infty}^{\infty} f W_{\phi_{m, n}}(t, f) d f .
$$

When we use a Gaussian of variance $\sigma^{2}$ for the Gabor synthesis atom, closed-form expressions can be developed for the integrals in the summation. ${ }^{1}$ Applying the same expansion to the denominator, we can express (9) as

$$
v(t)=\frac{\sum_{m, n, m^{\prime}, n^{\prime}} c_{m, n} c_{m^{\prime}, n^{\prime}}^{*} V_{m, n, m^{\prime}, n^{\prime}}(t)}{\sum_{m, n, m^{\prime}, n^{\prime}} c_{m, n} c_{m^{\prime}, n^{\prime}}^{*} A_{m, n, m^{\prime}, n^{\prime}}(t)}
$$

where

$$
\begin{aligned}
A_{m, n, m^{\prime}, n^{\prime}}(t):= & \frac{1}{2 \pi} \exp \left[-j 2 \pi\left(n-n^{\prime}\right) F t\right] \\
& \times \exp \left[-\frac{(t-m T)^{2}+\left(t-m^{\prime} T\right)^{2}}{2 \sigma^{2}}\right]
\end{aligned}
$$

and

$$
\begin{aligned}
V_{m, n, m^{\prime}, n^{\prime}}(t):= & {\left[\frac{-j\left(m-m^{\prime}\right) T}{4 \pi \sigma^{2}}-\frac{\left(n+n^{\prime}\right) F}{2}\right] } \\
& \times A_{m, n, m^{\prime}, n^{\prime}}(t) .
\end{aligned}
$$

If all $\left(m, n, m^{\prime}, n^{\prime}\right)$ combinations are included in the estimate, then this formula is equivalent to the (high-variance) WD-based estimate. By adopting the hybrid TFR approach [retaining only a subset of the terms when $\left.(m, n) \neq\left(m^{\prime}, n^{\prime}\right)\right]$, we can reduce the variance with the introduction of some bias. The performance is of a level similar to that obtained with adaptive quadratic TFRs; however, the computational expense is much

\footnotetext{
${ }^{1}$ Similar analytic expressions are possible for other windows, including, for example, square, triangle, and raised cosine windows.
} 
reduced since the $A_{m, n, m^{\prime}, n^{\prime}}(t)$ and $V_{m, n, m^{\prime}, n^{\prime}}$ are signal-independent and can be precomputed and stored in memory. The primary cost of the algorithm is then that of computing the (barely oversampled) Gabor transform.

The second-order moment of the hybrid TFR provides a similarly computationally efficient estimate of the instantaneous bandwidth

$$
B^{2}(t)=\frac{\sum_{m, n, m^{\prime}, n^{\prime}} c_{m, n} c_{m^{\prime}, n^{\prime}}^{*} D_{m, n, m^{\prime}, n^{\prime}}(t)}{\sum_{m, n, m^{\prime}, n^{\prime}} c_{m, n} c_{m^{\prime}, n^{\prime}}^{*} A_{m, n, m^{\prime}, n^{\prime}}(t)}-v^{2}(t)
$$

with

$$
\begin{aligned}
D_{m, n, m^{\prime}, n^{\prime}}(t):= & \frac{1}{(4 \pi)^{2}} \exp \left[-\frac{(t-m T)^{2}+\left(t-m^{\prime} T\right)^{2}}{2 \sigma^{2}}\right] \\
& \times \exp \left[-j 2 \pi\left(n-n^{\prime}\right) F t\right] \\
& \times\left[-\frac{\left(T\left(m-m^{\prime}\right)\right)^{2}}{\sigma^{4}}\right. \\
& \quad+\frac{2+4 \pi j T F\left(m-m^{\prime}\right)\left(n+n^{\prime}\right)}{\sigma^{2}} \\
& \left.+4 \pi^{2}\left(F\left(n+n^{\prime}\right)\right)^{2}\right] .
\end{aligned}
$$

Fig. 2 shows a comparison between the instantaneous bandwidth measures computed using the spectrogram and the hybrid TFR approach. One hundred random bandpass signals were generated by applying a time-varying filter to white Gaussian noise realizations. Fig. 2(a) depicts the ideal frequency response of the filter. Fig. 2(b) plots two lines for each technique; the lines show the averages of $v(t) \pm B(t)$ over the 100 realizations. Clearly, the hybrid TFR bandwidth estimate tracks the true bandwidth characteristics of the signal far more closely than the spectrogram-based measure.

If the hybrid estimate if formed without cross-terms, then the performance is very similar to the spectrogram. We can provide no strict guidelines regarding the choice of the distance threshold. In our experiments, we have observed that Manhattan distance thresholds $\delta$ between 1 and 3 provide good results. Setting $\delta=1$ results in more smoothing and, hence, more robust estimates in very noisy environments. The choice $\delta=3$ reduces the bias of estimates and is appropriate when there is little noise and a single dominant signal component.

It is possible to extend this method to estimate signal attributes derived from higher order moments. In particular, we can estimate instantaneous skew and instantaneous kurtosis [4] in a similar fashion, but the third- and fourth-order terms generate more complicated expressions that we do not provide here.

\section{Application to Seismic Data Analysis}

As an example of the hybrid IF estimation procedure, we now consider an application in seismic data analysis. Seismic imagery of the earth's subsurface is critical to all aspects of the oil and gas exploration process-from the location of reserves to their appraisal and subsequent monitoring. A seismic image of the earth is obtained by probing the subsurface with acoustic waves. An example of a seismic cross section is shown in Fig. 3(a). The horizontal axis gives the spatial location at the
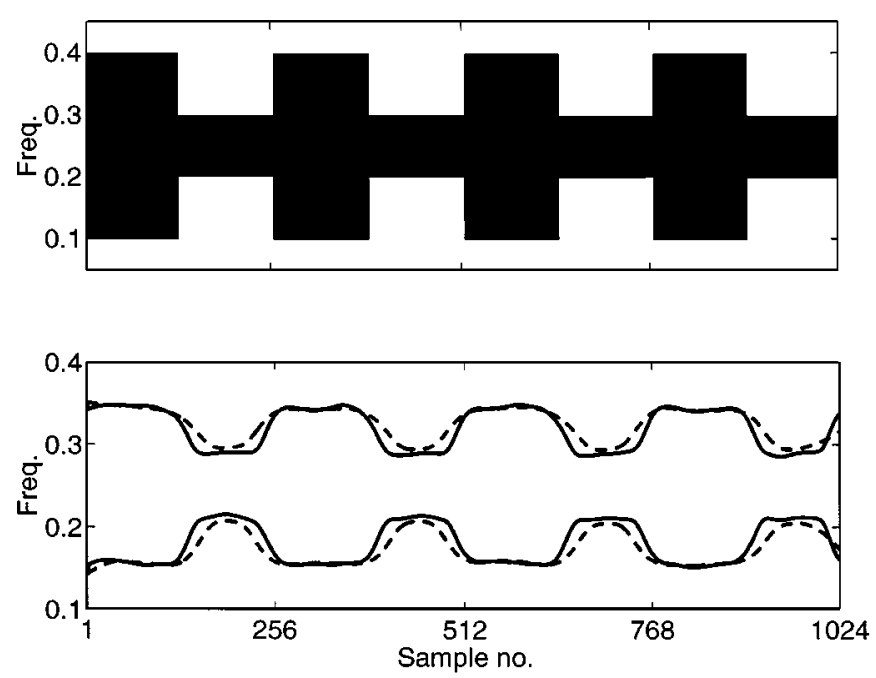

Fig. 2. Comparison between instantaneous bandwidth measures computed using the hybrid TFR approach and the spectrogram. White Gaussian noise was passed through a time-varying filter with (ideally) the frequency response in the top panel to generate 100 random realizations. The passband is indicated by the dark region. The lines in the bottom panel correspond to the averaged estimated IF \pm the averaged estimated bandwidth $(v(t) \pm B(t))$ calculated using the hybrid approach with Manhattan distance threshold $\delta=2$ (solid) and the spectrogram moments (dashed).

surface, and the vertical axis is time. Each column of the image represents a recording of the pressure as a function of time at the corresponding surface location. The line plot to the left of the intensity image is the amplitude of the first column [like Fig. 1(a)]. When seismic waves propagate through the subsurface, a part of their energy is reflected back to the surface at acoustic impedance contrasts. The strength of this impedance contrast is called the reflectivity. The seismic cross section represents this (bandlimited) reflectivity of the subsurface.

A change in acoustic impedance indicates a change in rock properties. As a result, it is possible to make an educated guess of the geological structure of the subsurface on the basis of the reflectivity image. If the seismic wave velocity is known, then the vertical time axis can be converted to depth. In the cross section of Fig. 3(a), a 1-s two-way travel time of the seismic waves corresponds to a depth of approximately $1 \mathrm{~km}$. The reflectivity image is obtained from the raw data through a great number of data processing steps. The most important of these are source deconvolution, noise suppression, velocity correction, and a final imaging step (migration) [16].

In seismic exploration, seismic cross sections are scrutinized by interpreters who search for features that indicate hydrocarbon reservoirs. While previously intepreters dealt with large plots of 2-D cross sections, they now work on computers with $3-D$ volumes comprising many gigabytes of data. Currently, there is a great need for methods that can aid in rapidly sifting out those features that are relevant for the geological interpretation of the data.

Seismic attributes aid the interpretation of seismic data by elucidating its salient signal characteristics. Traditionally, complex-trace analysis via Hilbert transform [using (8)] has been used for attribute extraction [17]. The standard seismic attributes are instantaneous amplitude, phase, and frequency. The goal of seismo-stratigraphic interpretation is to infer the sequence of geological events that resulted in the reflection pattern observed 
(a)

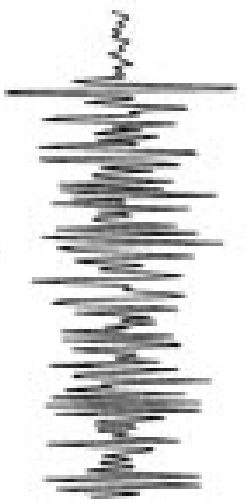

(b)

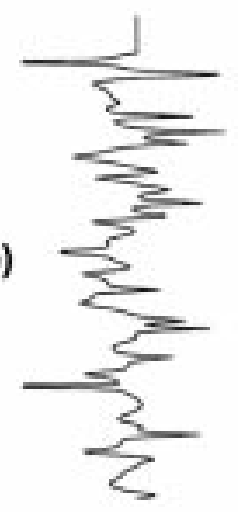

(c)

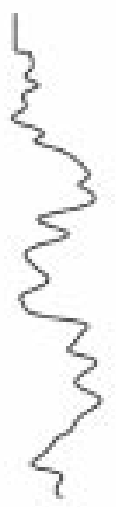

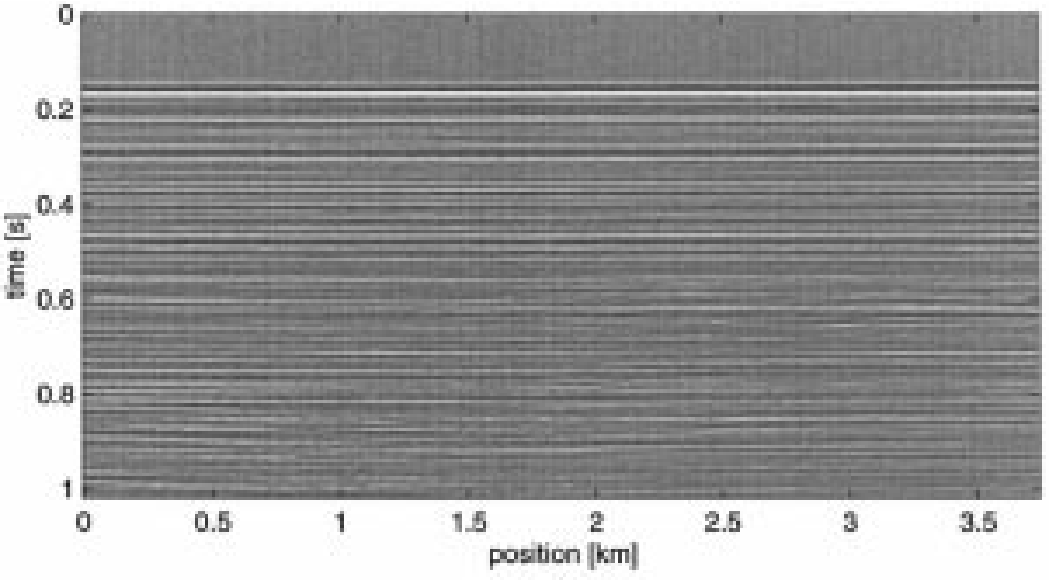
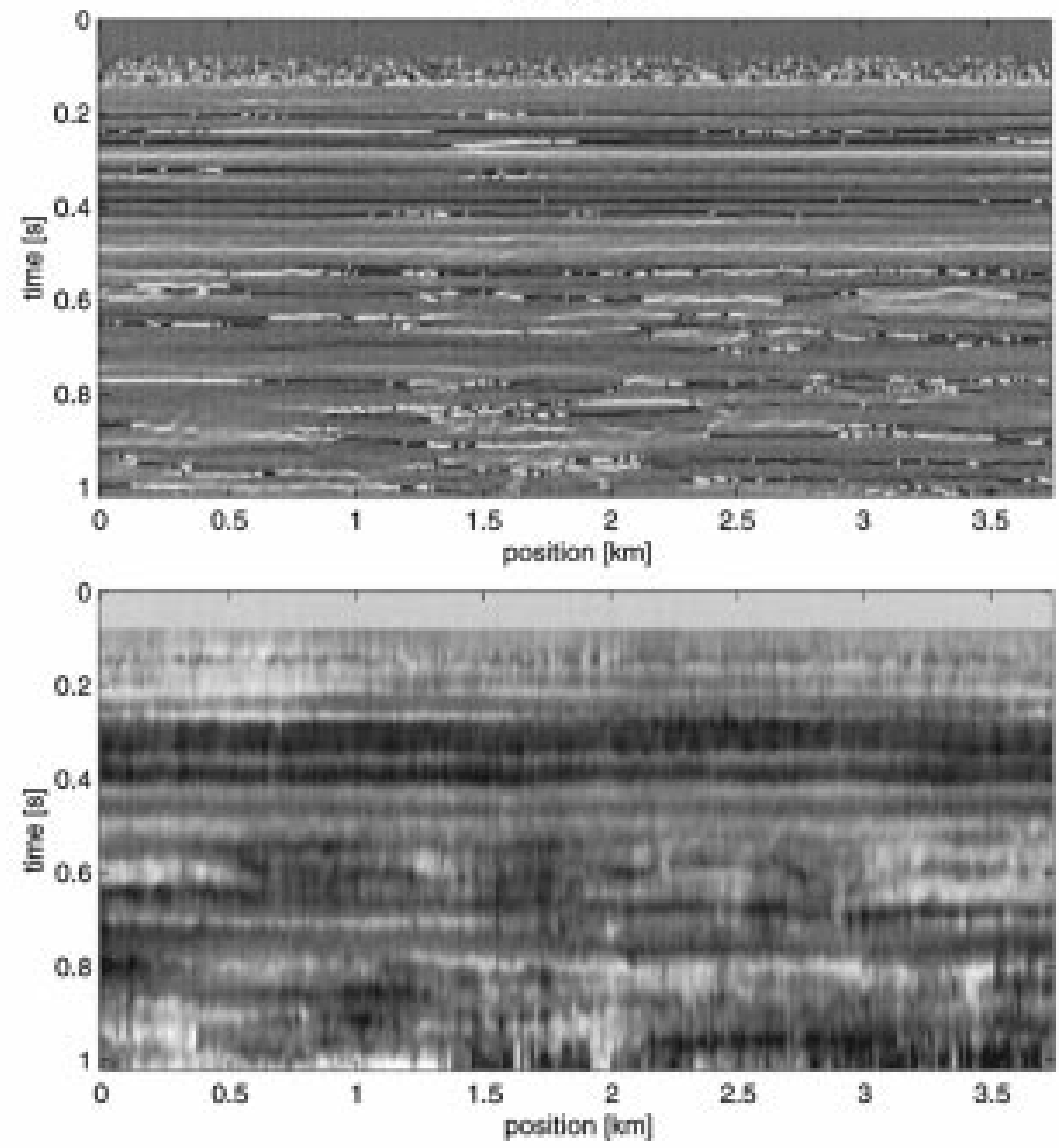

Fig. 3. Example of 2-D seismic analysis. (a) The 2-D seismic cross section. Trace to the left shows the amplitude of the first column of the cross section [like Fig. 1(a)]. (b) Complex-trace IF estimates (8) of each column of the cross section displayed as an intensity map (black corresponds to high frequencies and white to low frequencies). Trace to the left shows the complex-trace IF estimate of first column. (c) Intensity map of hybrid TFR IF estimates (12) of the columns of the cross section (Manhattan distance threshold $\delta=2$ ). Trace to the left shows the hybrid TFR IF estimate of first column.

in a seismic image [18]. In the ideal case, the seismic expression of a rock formation can be directly related to the circumstances under which the rock was formed. A seismic reflection pattern with a distinct set of image characteristics is called a seismic facies unit. For instance, rapid sedimentation in a high-energy environment (rivers or channels, for example) often produces strong lateral changes in reflection characteristics, whereas slow deep sea sedimentation usually results in seismic facies that consist of a laterally continuous reaction pattern.

The first step in a seismic interpretation is the delineation and description of the different seismic facies units present. The role of seismic attributes in seismic stratigraphy is to aid in delineating seismic facies units and in quantifying seismic facies descriptions. For instance, reaction continuity can be quantified by measuring the degree of lateral change in reflectivity and IF.

Another application of seismic attribute analysis is the prediction of physical rock properties such as porosity or permeability from seismic data. Ground truth is provided by measurement in a borehole. Lateral prediction away from the well is then obtained by correlating the physical parameter with the seismic attributes. In this application, the seismic attributes provide the input for a multivariate statistical analysis (using a neural network, for example). 
In both the visual interpretation and the statistical pattern analysis applications, robust attribute extraction methods are of crucial importance. IF brings forward those characteristics from the image that in the standard image can be obscured by the large dynamic range in amplitude. Subtle changes in the spacing of reflectors are not neccesarily visible in the standard amplitude cross section [see Fig. 3(a), for example] but will be brought forward in an IF display.

IF estimates based on TFR peaks do not exhibit sufficient continuity in space to aid interpretation of the data. IF estimates based on the moments of the TFR provide a much more informative portrayal of the data [3], [19]. Fig. 1 compares the IF estimates from three different TFRs on a seismic time signal. The fast hybrid IF estimate is very close to that obtained using the much more computationally expensive AOK TFR [3], [19]. The time resolution of the spectrogram IF estimate is significantly poorer than the other two methods. Moreover, a substantial smoothing is noticeable where rapid changes occur (at $t=0.4 \mathrm{~s}$, for example). Accurate localization in time of these rapid changes is important in seismic interpretation, as they may indicate important seismic facies sequence boundaries, which in turn may represent an important change in geological environment.

Fig. 3(b) and (c) show complex-trace IF (8) and hybrid IF estimates (12) of the columns of the seismic section of Fig. 3(a). To the left of the images, we plot the amplitude of the left-most column. The wild fluctuations indicate that the complex-trace IF estimate is very noise sensitive. The sharp peaks in the complex-trace IF estimate obscure the frequency trends of interest for a seismic facies analysis. Closely spaced seismic reflectors manifest themselves as bands of relatively high IF. The band of high-frequency reflections at $t=0.4 \mathrm{~s}$ clearly stands out in the hybrid IF section, whereas it is difficult to localize in the amplitude display of Fig. 3(a). Indeed, the abrupt change at $t=0.4 \mathrm{~s}$ coincides with an important geological boundary. Lateral changes in reflector spacing and frequency are also more easily detected in the IF cross section than in the amplitude data. For example, the reaction pattern below $t=0.4 \mathrm{~s}$ exhibits less lateral continuity than the upper part of the section, indicating a different depositional environment.

Hybrid IF estimation is a computationally very efficient method for seismic attribute extraction. For visual interpretation, the hybrid IF provides a greatly enhanced image as compared with the complex-trace IF or the spectrogram average. The estimation algorithm potentiates the TFR-based interpretation of large seismic data volumes, which up to now has been severely inhibited by the large computational effort involved in calculating TFRs.

\section{CONCLUSIONS}

We have presented a robust and efficient method for estimating time-frequency attributes of a signal. Using hybrid linear/quadratic time-frequency concepts avoids the expensive calculation of a quadratic or adaptive TFR. While we have focused on estimating the IF and instantaneous bandwidth, closed-form expressions analogous to (9) can be developed for many other attributes, including higher order time-frequency moments, kurtosis [4], and so on. Our results have particular relevance for seismic data analysis, where vast volumes of data require processing, and it is critical that efficient algorithms be used.

\section{REFERENCES}

[1] B. Boashash, "Estimating and interpreting the instantaneous frequency of a signal-Part I: Fundamentals-Part II: Algorithms," Proc. IEEE, vol. 80, pp. 519-569, Apr. 1992.

[2] S. Haykin, R. Racine, Y. Xu, and C. Chapman, "Monitoring neuronal oscillations and signal transmissions between cortical regions using time-frequency analysis of electroencephalographic activity," Proc. IEEE, vol. 84, pp. 1295-1301, 1996.

[3] P. Steeghs and G. Drijkoningen, "Sequence analysis and attribute extraction with quadratic time-frequency representations,", submitted for publication.

[4] P. Loughlin and K. Davidson, "Instantaneous kurtosis," IEEE Signal Processing Lett., vol. 7, pp. 156-159, 2000.

[5] B. Boashash and P. O'Sheah, "Use of cross Wigner-Ville distribution for estimation of instantaneous frequency," IEEE Trans. Signal Processing, vol. 41, pp. 1439-1445, Mar. 1993.

[6] F. Çakrak and P. Loughlin, "Instantaneous frequency estimation of polynomial phase signals," in Proc. IEEE Symp. Time-Freq. Time-Scale Anal., 1998, pp. 549-552.

[7] B. Ristic and B. Boashash, "Instantaneous frequency estimation of quadratic and cubic FM signals using the cross polynomial Wigner-Ville distribution," IEEE Trans. Signal Processing, vol. 44, pp. 1549-1553, June 1993.

[8] S. Qian and J. Morris, "Wigner distribution decomposition and crossterms deleted representation," Signal Process., vol. 27, pp. 125-144, May 1992.

[9] S. Qian and D. Chen, "Decomposition of the Wigner distribution and time-frequency distribution series," IEEE Trans. Signal Processing, vol. 42, pp. 2836-2842, Oct. 1994.

[10] M. Pasquier, P. Gonçalves, and R. Baraniuk, "Hybrid linear/bilinear time-scale analysis," IEEE Trans. Signal Processing, vol. 47, pp. 254-259, Jan. 1999.

[11] D. Gabor, "Theory of communication," J. Inst. Elect. Eng., vol. 93, pp. 429-444, 1946.

[12] L. Cohen, Time-Frequency Analysis. Englewood Cliffs, NJ: PrenticeHall, 1995.

[13] D. Jones and R. Baraniuk, "An adaptive optimal-kernel time-frequency representation," IEEE Trans. Signal Processing, vol. 43, pp. 2361-2371, Oct. 1995.

[14] L. Cohen and C. Lee, "Instantaneous quantities, standard deviation and cross-terms," in Proc. SPIE Conf. Adv. Alg. Arch. Signal Process. III, vol. 975, San Diego, CA, July 1988, pp. 186-208.

[15] P. Flandrin, Time-Frequency and Time-Scale Analysis. San Diego, CA: Academic, 1999.

[16] O. Yilmaz, Seismic Data Processing. Tulsa, OK: Soc. Explor. Geophys., 1987.

[17] T. Taner, F. Koehler, and R. Sheriff, "Complex seismic trace analysis," Geophys., vol. 44, pp. 1041-1063, 1979.

[18] C. Payton, Ed., AAPG Memoir, ch. Seismic Stratigraph-Applications to Hydrocarbon Exploration. Tulsa, OK: Amer. Assoc. Petrol. Geol., 1976, pp. 301-328.

[19] P. Steeghs and G. Drijkoningen, "Time-frequency analysis of seismic sequences," in Proc. 65th SEG Meet., Houston, TX, 1995.

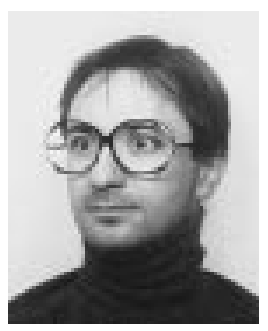

Richard G. Baraniuk (SM'98) received the B.Sc. degree in 1987 from the University of Manitoba, Winnipeg, Canada, the M.Sc. degree in 1988 from the University of Wisconsin-Madison, and the Ph.D. degree in 1992 from the University of Illinois, Urbana-Champaign, all in electrical engineering.

In 1986, he was a Research Engineer with Omron Tateisi Electronics, Kyoto, Japan. After spending 1992 and 1993 with the Signal Processing Laboratory, Ecole Normale Superieure, Lyon, France, he joined Rice University, Houston, TX, where he is currently a Professor of Electrical and Computer Engineering and a DJ at KTRU. He spent Autumn 1998 at the Isaac Newton Institute, Cambridge University, Cambridge, U.K., as the Rosenbaum Fellow. His research interests lie in the area of signal and image processing and include wavelets, probabilistic models, networks, and time-frequency analysis. He serves on the editorial board of the journal Applied and Computational Harmonic Analysis. 
Dr. Baraniuk received a NATO Postdoctoral Fellowship from NSERC in 1992, the National Young Investigator award from the National Science Foundation in 1994, a Young Investigator Award from the Office of Naval Research in 1995, the Rosenbaum Fellowship from the Newton Institute in 1998, the C. Holmes MacDonald National Outstanding Teaching Award from Eta Kappa Nu in 1999, the Charles Duncan Junior Faculty Achievement Award from Rice University in 2000, and the ECE Young Achievement Award from the University of Illinois in 2000. He was a co-author on a paper with M. Crouse and R. Nowak that received the IEEE Signal Processing Society Junior Paper Award in 2001. He is a member of the "Signal Processing Theory and Methods" Technical Committee of the IEEE Signal Processing Society.

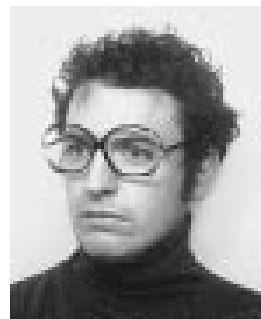

Mark Coates (M'99) received the B.E. degree in computer systems in 1995 from the University of Adelaide, Adelaide, Australia, and the Ph.D. degree in electrical engineering in 1999 from the University of Cambridge, Cambridge, U.K.

In 2000, he was the Texas Instruments Postdoctoral Fellow at Rice University, where he is currently a Research Associate and Lecturer. His research interests lie in the area of statistical signal processing, including network modeling and inference, sequential Monte Carlo methods, and time-frequency anal-

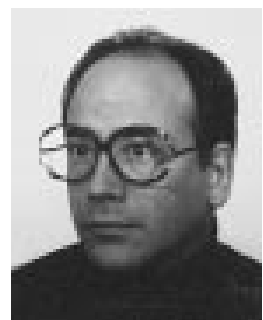

Philippe Steeghs received the M.Sc. degree in exploration geophysics in 1992 from Utrecht University, Utrecht, The Netherlands, and the Ph.D. from Delft University of Technology, Delft, The Netherlands, in 1997.

From 1997 to 1999 , he was a Postdoctoral Fellow in electrical and computer engineering at Rice University, Houston, TX, and a Principal Investigator with the Rice Conosortium on Computational Seismic Interpretation. He is currently a Senior Scientist with the Radar and Signal Processing Group, Dutch Organization for Applied Scientific Research (TNO), The Hague, The Netherlands. His research interests are in time-frequency/time-scale methods, seismic data processsing and interpretation, and radar signal processing.

ysis. 